\title{
Visão de enfermeiras sobre as articulações das ações de saúde entre profissionais de equipes de saúde da família*
}

\author{
NURSES' VIEW ON THE COORDINATION OF HEALTH ACTIONS \\ AMONG PROFESSIONALS OF FAMILY HEALTH TEAMS
}

VISION DE ENFERMERAS ACERCA DE LAS ARTICULACIONES DE LASACCIONES
DE SALUD ENTRE PROFESIONALES DE EQUIPOS DE SALUD DE LA FAMILIA

Isabel Cristina dos Santos Colomé ${ }^{1}$, Maria Alice Dias da Silva Lima², Roberta Davis ${ }^{3}$

\section{RESUMO}

Este estudo objetivou conhecer a concepção de enfermeiras que atuam no Programa de Saúde da Família sobre o desenvolvimento do trabalho em equipe, no que diz respeito à articulação das ações dos diversos profissionais que a compõem. Trata-se de um estudo descritivo de abordagem qualitativa. A coleta de dados foi realizada por meio de entrevistas semiestruturadas com 23 enfermeiras. A análise dos dados foi realizada com base na Análise Temática. Os resultados mostraram que as enfermeiras, em alguns momentos, articulam suas ações com os demais profissionais da equipe. Entretanto, existem fatores que dificultam essa articulação como o excesso de demanda de usuários, a falta de tempo dos profissionais para realizar o planejamento coletivo de suas práticas e desenvolver ações preventivas. Conforme os resultados obtidos, torna-se necessária a reorganização do processo de trabalho, com intenções da realização de um trabalho efetivamente integrado.

\section{DESCRITORES}

Programa Saúde da Família. Relações interprofissionais. Equipe de assistência ao paciente/ organização \& administração.

\begin{abstract}
This study was aimed at knowing the conception nurses who work in the Family Health Program have about the development of teamwork regarding the coordination of the actions of the various professionals that comprise it. It is a descriptive study with a qualitative approach. The data collection was carried out through semi-structured interviews with 23 nurses. The analysis of the data was carried out based on Theme Analysis. The results showed that the nurses, at certain moments, coordinate their actions with the other professionals of the team. However, there are factors that make this coordination difficult, such as excess of demand from users and lack of time for the professionals to prepare the collective planning of their practices and develop preventive actions. According to the results obtained, it is necessary to reorganize the work process in order to perform an effectively integrated work.
\end{abstract}

\section{KEY WORDS}

Family Health Program. Interprofessional relations. Patient care team/ organization \& administration.

\section{RESUMEN}

Este estudio tuvo por objetivo conocer la concepción de enfermeras, que actúan en el Programa de Salud de la Familia, respecto al desarrollo del trabajo en equipo, en cuanto a la articulación de las acciones de los diversos profesionales que lo componen. Se trata de un estudio descriptivo de abordaje cualitativo. La recolección de datos fue realizada por medio de entrevistas semi-estructuradas a 23 enfermeras. El análisis de los datos se hizo con base en el Análisis Temático. Los resultados mostraron que las enfermeras, en algunos momentos, articulan sus acciones con los demás profesionales del equipo. No obstante, existen factores que dificultan esa articulación como el exceso de demanda de usuarios, la falta de tiempo de los profesionales para realizar la planificación colectiva de sus prácticas y desarrollar acciones preventivas. De acuerdo a los resultados obtenidos, se torna necesaria la reorganización del proceso de trabajo, con el objetivo de realizar un trabajo efectivamente integrado.

\section{DESCRIPTORES}

Programa Salud de la Familia. Relaciones interprofesionales. Grupo de atención al paciente/ organización \& administración.

\footnotetext{
* Extraído da dissertação "Trabalho em equipe no Programa Saúde da Família na concepção de enfermeiras", Escola de Enfermagem, Universidade Federal do Rio Grande do Sul (UFRGS), 2005. ${ }^{1}$ Mestre em Enfermagem pela UFRGS. Professora Assistente do Curso de Enfermagem da Universidade Federal de Santa Maria (UFSM), Centro de Educação Superior Norte (CESNORS) de Palmeira das Missões. Palmeira das Missões, RS, Brasil. enfbel@yahoo.com.br 2 Professora Associada da Escola de Enfermagem da UFRGS. Doutora em Enfermagem pela Universidade de São Paulo (USP). Porto Alegre, RS, Brasil. malice@enf.ufrgs.br ${ }^{3}$ Enfermeira do Setor de Emergência do Hospital Nossa Senhora da Conceição de Porto Alegre. Porto Alegre, RS, Brasil. betadavis@hotmail.com
} 


\section{INTRODUÇÃO}

0 trabalho em saúde contempla a esfera não-material, sendo que seu produto consiste na própria prestação da assistência de saúde, a qual é produzida ao mesmo tempo em que é consumida ${ }^{(1-2)}$. Além disso, está inserido na racionalidade de uma dada sociedade, tendo suas funções determinadas por necessidades sociais, atendendo a finalidades individuais e coletivas ${ }^{(3)}$.

Para a realização do trabalho em saúde torna-se necessária a articulação das ações de diversos profissionais( ${ }^{(4-5)}$. Sobretudo no que se refere ao Programa de Saúde da Família (PSF), essa premissa torna-se indispensável, sendo as equipes multiprofissionais consideradas um dos instrumentos fundamentais para o desenvolvimento do trabalho e consolidação dos objetivos do Programa(6).

No entanto, um dos grandes desafios que se apresenta aos profissionais de saúde que vêm atuando no âmbito da saúde da família refere-se à integração da equipe de trabalho. Se essa dificuldade não for superada, é possível que haja a repetição de um modelo de atenção à saúde fragmentado, voltado ao enfoque da recuperação biológica individual, rígido na divisão do trabalho e desigual no reconhecimento social dos diversos trabalhos ${ }^{(7)}$.

Em estudo realizado em um município do Estado do Rio de Janeiro foi identificado que há falta de coesão no desenvolvimento do trabalho no PSF, tornando-se evidentes as dificuldades da equipe em atuar de forma integrada ${ }^{(8)}$.

Ressalta-se que trabalhar em equipe no PSF de maneira integrada significa estabelecer conexões entre os distintos processos de trabalho, fundamentando-se em um certo conhecimento sobre o trabalho do outro e na valorização das contribuições deste na produção de cuidados. 0 estabelecimento de consensos entre os profissionais, no que se refere aos objetivos e resultados a serem contemplados e à melhor forma de atingi-los, também caracteriza um trabalho integrado(9).

Existem duas dimensões inerentes ao trabalho em equipe, sendo que uma diz respeito à interação dos profissionais e a outra se refere à articulação das ações, a qual pode ser definida como momentos em que os agentes, ativamente, colocam em evidência as conexões ou nexos existentes entre as distintas ações e os variados saberes técnicos( ${ }^{(4)}$.

Nesta perspectiva, destaca-se a necessidade do desenvolvimento de um trabalho conjunto, no qual todos os profissionais envolvam-se em algum momento da assistência e, agindo de acordo com seu nível de competência específico, formem um saber capaz de dar conta da com- plexidade dos problemas e necessidades de saúde dos indivíduos e da coletividade.

Assim, tem-se como objetivo conhecer a concepção de enfermeiras que atuam no Programa Saúde da Família sobre o desenvolvimento do trabalho em equipe, no que se refere à articulação das ações realizadas pelos diversos profissionais.

\section{MÉTODO}

0 estudo teve como referencial teórico-metodológico a pesquisa qualitativa, que permite 0 aprofundamento no mundo dos significados, relações humanas, atitudes, crenças e valores ${ }^{(10)}$

Os sujeitos do estudo foram 23 enfermeiras que compõem equipes atuantes em Unidades Básicas de Saúde com Programa Saúde da Família no município de Porto Alegre, Rio Grande do Sul.

A coleta de dados foi realizada por meio de entrevistas semi-estruturadas. Foi utilizado gravador a fim de garantir a fidedignidade do registro das respostas. Posteriormente, as fitas gravadas foram transcritas na íntegra.

Para análise dos dados foi utilizada a técnica de Análise Temática(11). Os dados foram classificados e agregados, estabelecendo-se três categorias empíricas: (1) Concepções das enfermeiras sobre o trabalho em equipe; (2) Articulação das ações dos profissionais no trabalho em equipe; (3) Interação da equipe. Neste artigo, são apresentados os resultados referentes à segunda categoria.

0 projeto de pesquisa foi aprovado pelo Comitê de Ética em Pesquisa da Secretaria Municipal de Saúde de Porto Alegre (Parecer Projeto 286/2004). As enfermeiras receberam e assinaram o Termo de Consentimento Livre e Esclarecido, o qual garantiu o anonimato e o caráter confidencial das informações, de acordo com a Resolução $196 / 96$ do Conselho Nacional de Saúde ${ }^{(12)}$. Para preservar o anonimato, cada entrevistada foi identificada com um código (ENF1, ENF2, ENF3, ENF4...), de acordo com a ordem cronológica de realização das entrevistas.

\section{RESULTADOS E DISCUSSÃO}

A articulação das ações ocorre nos momentos em que os diferentes profissionais da equipe evidenciam conexões entre os diferentes trabalhos ${ }^{(4-5)}$. Considera-se que haja articulação nas situações em que os diversos profissionais promovam troca de idéias e informações sobre 0 trabalho, discussão de casos e tomada de decisões em equipe, utilizando as diferentes competências e funções 
para a busca conjunta de resolução para os problemas de saúde dos usuários.

As enfermeiras mencionaram que a articulação das ações se estabelece principalmente com os agentes comunitários de saúde. Isso se explica pelo fato de essas profissionais serem responsáveis pela supervisão do trabalho dos agentes e estarem mais próximas deles no diaa-dia de sua prática. Assim, ficaram evidentes as atribuições dos agentes comunitários de realizarem a busca ativa dos usuários faltosos e transmitirem as informações à enfermeira. Os dados trazidos por essas profissionais são fundamentais para que a enfermeira mantenha o controle da situação das famílias sob sua responsabilidade, como evidencia o seguinte depoimento:

\section{[...] eu já deixei dois casos separados e que as agentes comunitárias também já estão sabendo, então a gente vai fazendo essa troca. Quando eu atendo uma criança que eu vejo que está com as vacinas atrasadas, primeira coisa que eu vou querer saber, vou lá falar com a agente [...] (ENF9).}

Os agentes comunitários possuem informações importantes que subsidiam o trabalho da enfermeira e dos demais componentes da equipe. Estudo revelou que, na ótica dos profissionais de saúde atuantes no PSF, o papel fundamental do agente comunitário é servir de elo entre a equipe de saúde e a comunidade. Essa ligação constrói-se a partir da troca de informações que esse profissional estabelece com ambos os lados. Todavia, salienta-se que 0 agente não pode constituir um elo isolado ligando duas partes, sem, contudo, interagir nessa corrente: ele deve promover uma verdadeira integração com os profissionais de saúde e a comunidade(13).

Constata-se que as enfermeiras valorizam o trabalho realizado pelos agentes de saúde, justamente pelo contato direto que estes mantêm com as famílias, o que viabiliza o conhecimento da equipe sobre a realidade das mesmas, como se pode identificar a seguir:

[...] os agentes comunitários têm um contato direto com as famílias [...] conhecem detalhes das famílias que de repente numa consulta aqui no consultório tu não vai ter acesso de jeito nenhum, nem o enfermeiro, nem o médico. Então muitas coisas tu recompõe, tu analisa de uma outra forma com esse conhecimento que o agente traz da rua [...] (ENF14).

Verificou-se que as enfermeiras exercem uma atividade de supervisão e liderança do trabalho realizado pelos agentes comunitários e auxiliares de enfermagem, servindo de referência para esses profissionais. Assim, devem procurar auxiliar na identificação, análise e resolução dos problemas que lhe são apresentados.

A primeira categoria de classe que eles procuram, tanto auxiliar como agente de saúde, é sempre o enfermeiro. [...] a gente é muito procurada para resolver tudo. [...] Quando eles vêm das visitas domiciliares, cada um já vem com um bloquinho de problemas e que tu tem que tentar solucionar (ENF21).
As enfermeiras também conectam suas ações com as de outros profissionais da equipe, como dentista e médico, determinando, assim, a articulação com o trabalho de todos os profissionais da equipe.

[...] atendo uma criança que tu vai examinar a cavidade oral e vê que está um caos a boquinha; daí já dou ali para a dentista, já converso com ela [...] muitas vezes tu faz uma ausculta pulmonar e vê que está bem alterado; já vou lá e já peço para ele [médico]: Olha, dá para ir lá na sala ver o nenê? Já discuto o caso com ele; já vem aqui, já examina; a criança já sai com a prescrição médica daqui do consultório [...] (ENF10).

Esse depoimento remete ao caráter complementar dos diferentes trabalhos, no momento em que a enfermeira aciona outros profissionais para intervirem em uma necessidade do usuário, a qual está fora de sua capacidade resolutiva. Dessa forma, pode-se inferir que a enfermeira está comprometida em resolver os problemas, pois realiza os encaminhamentos necessários imediatamente a fim de evitar que o usuário precise retornar ao serviço em um outro dia. Além disso, evidencia-se que um profissional precisa do trabalho do outro para dar continuidade às suas intervenções e para promover o desenvolvimento da assistência integral em saúde.

Em pesquisa realizada junto a uma equipe do PSF de um município baiano, com o objetivo de identificar evidências de articulação das ações e interação dos profissionais na equipe, verificou-se que, em diversos momentos do trabalho, os profissionais estabeleciam conexões entre as diferentes ações, buscando os demais membros da equipe para trocarem informações e esclarecerem dúvidas. Esses achados vêm ao encontro dos evidenciados neste estudo e caracterizam, portanto, a articulação entre as diversas práticas ${ }^{(14)}$.

Observa-se a complementaridade de ações no que se refere à utilização do prontuário da família, evidente no depoimento a seguir:

O prontuário é extremamente respeitado por todos aqui. Então tu vai pegar um prontuário nosso, eu tenho doutorando, técnico de enfermagem, enfermeiro, médico, todos seguindo. [...] eles vão seguir o que eu já tinha posto ali. Não vão fazer uma outra anamnese: eu já fiz a minha parte, eles vão complementa (ENF6).

O fato do prontuário de atendimento ser o mesmo para utilização de todos os membros da equipe permite que os profissionais tenham uma visão mais abrangente do histórico e do estado de saúde dos usuários, e, assim, complementem os demais trabalhos.

As enfermeiras apresentam como um fator de dificuldade o excesso de demanda de usuários pelo serviço, que ocasiona falta de tempo para articular os diversos trabaIhos e planejar as ações a serem desenvolvidas. Muitas vezes, sentem-se meras cumpridoras de tarefas, pois não encontram tempo para pensar sobre o seu fazer. 
[...] o planejamento nosso que está prejudicado. Eu acho que é isso que a gente teria que se organizar mais: a gente só faz, faz, faz e não planeja muito. Eu me sinto uma tarefeira, sem conseguir planejar as coisas, tu está sempre atendendo naquele ritmo acelerado e tu não pára para pensar como é que tu vai fazer tal coisa, como é que tu vai melhora. (ENF11).

Essa situação faz com que a equipe atue sobre casos isolados, de acordo com a demanda de usuários, pois há pouco tempo para planejar e implementar ações coletivas que visem à prevenção e à promoção da saúde da comunidade. A impossibilidade ou a dificuldade de se estabelecer metas e objetivos a serem alcançados provoca um sentimento de frustração na equipe, fator limitante na execução do trabalho.

Outra investigação realizada com uma equipe de saúde da família revelou que os momentos de articulação das ações entre os profissionais dão-se de maneira limitada, pois estão sempre voltados para a resolução de situações imediatas do cotidiano do trabalho provenientes das queixas dos usuários. Os autores afirmam que esse fato compromete a construção de estratégias preventivas referentes às necessidades da comunidade local ${ }^{(14)}$.

Com isso, questiona-se, dessa forma, se a estratégia saúde da família não estaria contribuindo para a manutenção de um modelo de atenção à saúde individual e biologicista, o qual desconsidera os inúmeros aspectos do contexto em que as famílias estão inseridas. 0 desenvolvimento de um trabalho parcelar, no qual cada profissional realiza suas atividades de forma desarticulada e independente das ações dos demais, pode contribuir, significativamente, para a falta de comprometimento e responsabilização desses agentes com 0 resultado de suas práticas.

Nessa perspectiva, destacam-se os conceitos de núcleo de competência e de responsabilidade e de campo de competência e de responsabilidade. 0 núcleo é definido como o conjunto de saberes e de responsabilidades específicos a cada profissão ou especialidade ${ }^{(15)}$. Portanto, pode ser entendido como o ponto de diferenciação entre as diversas profissões, no qual são evidenciadas as competências e os saberes particulares de cada uma. Já campo refere-se aos saberes e responsabilidades comuns ou confluentes a várias profissões ou especialidades ${ }^{(15)}$. Assim, conhecimentos básicos sobre o processo saúde-doença são comuns a todas as profissões da área da saúde, sendo, portanto um ponto de convergência entre elas.

As discussões acerca do núcleo de competência e de responsabilidade trazem a idéia de autonomia profissional, na medida em que se referem às especificidades dos saberes de cada profissional, mas também remetem à interdependência dessas autonomias. Neste sentido, o núcleo de competência de cada profissional, de maneira isolada, não é suficiente para contemplar a complexidade do atendimento das necessidades de saúde, sendo ne- cessário flexibilizar os limites das competências para garantir uma prática integral ${ }^{(7)}$.

As enfermeiras referiram que encontram dificuldades de tempo inclusive para se reunirem com as auxiliares de enfermagem, pois estas também possuem expressiva demanda de trabalho.

[...] Eles [auxiliares] estão com uma demanda muito grande de atividades [...] Então não tem como eu chegar e dizer Olha, tu tem que fazer isso, tu tem que trabalhar prevenção! Eles não têm como fazer. Eles têm que trabalhar em cima da demanda, e talvez isso dificulte a articulação [...] (ENF23).

As enfermeiras atribuíram o excesso de demanda de usuários ao número elevado de famílias designadas para cada equipe, inclusive em função da proposta de acolhimento desenvolvida pela Secretaria Municipal de Saúde (SM S) de Porto Alegre.

No ano de 2001, a SM S lançou o Projeto de Qualificação do Acolhimento na Atenção Básica, motivada pela necessidade de desenvolver ações que efetivassem os princípios do SUS, sendo o acolhimento um aspecto fundamental nesse sentido. Desta forma, o acolhimento foi definido como um processo no qual os trabalhadores de saúde e a organização assumem a responsabilidade de realizar intervenções na realidade presente em sua área de atuação, e, a partir da identificação das principais necessidades de saúde, buscam promover a saúde com base no estabelecimento de relações humanizadas e acoIhedoras, no âmbito individual e coletivo. Objetiva-se, ainda, a autonomia do usuário por meio de ações da equipe de saúde, da organização do setor saúde e de ações intersetoriais e interinstitucionais ${ }^{(16)}$.

Na equipe em que está implantado o acolhimento qualificado, há agenda aberta, ou seja, não existem dias e horários fixos para agendamento e é realizada a escuta de $100 \%$ dos usuários para avaliação de suas necessidades ${ }^{(16)}$. Nesse caso, todos os usuários que chegam ao serviço devem ser ouvidos e, a partir da avaliação de suas necessidades, encaminhados para as atividades oferecidas dentro da Unidade ou para outros serviços, conforme as particularidades de cada caso.

\footnotetext{
[...] com a questão do acolhimento, aumentou muito a demanda pelo serviço [...] Antes a gente fazia uma triagem para ver o que ia passar no dia; mas aquilo que não ia passar para consulta no dia não ia nem marcar nem nada: a pessoa ia sair daqui tendo que voltar um outro dia para marcar consulta. Então no ponto de vista do usuário facilitou muito; agora para nós é uma coisa que aumentou muito o trabalho que a gente tem [...] (ENF11).
}

Outra pesquisa evidenciou que os profissionais atuantes em unidades de saúde da família realizam um número elevado de acolhimentos diariamente. As autoras reconhecem que a prática de acolher amplia o acesso dos usuários aos serviços, porém gera a necessidade de dis- 
cussões por parte de profissionais e gestores sobre as demandas atendidas, a fim de reavaliar a oferta de serviços, os programas prioritários e a própria organização do trabalho da equipe ${ }^{(17)}$.

As enfermeiras salientaram que, com a atividade de acolhimento, a equipe fica sobrecarregada, pois deve realizar os encaminhamentos pertinentes de todos os usuários que chegam ao serviço. Além disso, algumas vezes, a população adscrita é maior do que a capacidade de atendimento da equipe.

Em investigação realizada com o objetivo de analisar a implantação do PSF no município de Florianópolis, os profissionais de saúde referiram que encontram inúmeros problemas relacionados à sobrecarga no dimen-sionamento das equipes, pois todas as Unidades estudadas possuem um número de famílias cadastradas superior ao previsto. Salientaram que isso ocasiona falta de tempo para a equipe realizar ações menos complexas, tais como visitas domiciliares e formação de grupos educativos ${ }^{(18)}$.

Dados semelhantes foram encontrados em outro estudo, o qual identificou que um dos fatores que dificultam a articulação das ações consiste no excesso de demanda que cada profissional da equipe absorve, decorrente do grande número de famílias cadastradas, o que prejudica a coesão da mesma e promove a fragmentação do trabalho ${ }^{(14)}$.

Em consonância com isso, considera-se que a sobrecarga de trabalho e a conseqüente falta de tempo são elementos que podem dificultar a articulação das ações na equipe, na medida em que os profissionais não encontram espaços no cotidiano para realizar interfaces e conexões entre os diversos saberes e práticas, desperdiçando, assim, o grande potencial do trabalho em equipe. Em contrapartida, nos questionamos se essa situação que resulta em excesso de atividades e falta de tempo não deriva justamente das dificuldades que as equipes possuem de realizar um trabalho integrado, pois a cooperação pode contribuir para a organização do trabalho e, conseqüentemente, para o seu melhor desenvolvimento.

Quando questionadas sobre a articulação entre as ações dos diversos profissionais, as entrevistadas referiram que ela ocorre principalmente no momento da reunião realizada semanalmente e está vinculada à necessidade da equipe de falar a mesma linguagem, isto é, afinar os discursos de forma que todos tenham o mesmo entendimento sobre determinados assuntos.

[...] a gente conseguiu preservar o espaço da reunião de equipe [...] esse é o principal momento de articulação e de combinações [...] (ENF1).

[...] nessas reuniões a gente coloca muito assim, sempre tem essa solicitação de que todos falem a mesma língua; porque eu não posso dar uma orientação para uma paciente, e depois vir a minha colega e dar uma orientação diferente (ENF15).
Em investigação ${ }^{(14)}$ junto a uma equipe do PSF, verificou-se que as reuniões ficavam restritas à busca de soluções para problemas administrativos, à divisão de tarefas entre os trabalhadores; à socialização de programações feitas pelos profissionais de nível superior; e a combinações sobre a dinâmica de atendimento na Unidade. Com isso, as reuniões não propiciavam a reflexão coletiva sobre o trabalho, dificultando o planejamento de ações conjuntas, já que estavam direcionadas basicamente para a transmissão de informações técnicas. Destaca-se a necessidade de ultrapassar esses limites, pois é fundamental que as reuniões proporcionem momentos para (re)pensar o cotidiano de trabalho e a interação entre os componentes da equipe, sendo um espaço que permita aos profissionais compartilhar seus anseios, dúvidas e expectativas. Ainda, entende-se que as reuniões devem permitir a discussão de problemas identificados e a construção de consensos, mesmo que temporários, sobre a resolução dos mesmos.

As enfermeiras encontraram limitações para realizar a articulação entre as diversas práticas, referindo que esta fica restrita basicamente aos momentos de reunião de equipe. Contudo, entende-se que essa conexão é inerente ao processo de trabalho diário dos profissionais e deve fazer parte da dinâmica da assistência, sem hora marcada para acontecer.

\section{CONSIDERAÇÕES FINAIS}

Constatou-se que, em diversos momentos, as enfermeiras conectam suas ações com as dos demais membros da equipe. Essa articulação é mais intensa com os agentes comunitários e os auxiliares de enfermagem, devido à função de supervisão que elas exercem junto a esses profissionais; mas também se estabelece com 0 médico e o dentista, normalmente nos momentos de atendimento aos usuários, com a finalidade de trocar informações, esclarecer dúvidas e realizar os encaminhamentos pertinentes.

A articulação das ações da equipe fica prejudicada pela excessiva demanda de usuários pelo serviço. Essa realidade acarreta sobrecarga de trabalho e falta de tempo para os profissionais planejarem em conjunto as ações a serem implementadas. Dessa forma, não conseguem programar e desenvolver ações preventivas, ficando envolvidos com 0 atendimento da demanda espontânea, geralmente realizado em suas salas individuais. Assim, tornam-se evidentes as limitações das enfermeiras para realizarem conexões entre os diversos trabalhos e interagirem no cotidiano de suas práticas, ficando essas questões restritas basicamente aos momentos de reunião de equipe.

Essa realidade exige a reorganização do processo de trabalho. Para isso, são necessárias discussões entre usuários, equipe e gestão municipal, visando à busca con- 
junta de soluções para o problema, na medida em que as dificuldades da equipe em realizar um trabalho efetivamente integrado interferem, sobremaneira, na qualidade da assistência prestada à população.

\section{REFERÊNCIAS}

1. Gonçalves RBM. Práticas de saúde: processo de trabaIho e necessidades. São Paulo: Secretaria Municipal de Saúde; 1992.

2. Pires D. Novas formas de organização do trabalho em saúde e enfermagem. Rev Baiana Enferm. 2000;13(1/2):8392.

3. Vaz MRC. Trabalho em saúde: expressão viva da vida social. In: Leopardi MT, organizador. O processo de trabalho em saúde: organização e subjetividade. Florianópolis: Papa-Livros; 1999. p. 57-70.

4. Peduzzi M. Equipe multiprofissional de saúde: a interface entre trabalho e interação [tese]. Campinas: Faculdade de Ciências Médicas, Universidade Estadual de Campinas; 1998.

5. Peduzzi M. Equipe multiprofissional de saúde: conceito e tipologia. Rev Saúde Pública. 2001;35(1):103-9.

6. Brasil. Ministério da Saúde. Secretaria de Políticas de Saúde. Departamento de Atenção Básica. Reunião dos coordenadores dos pólos de capacitação, formação e educação permanente em Saúde da Família: relatório final. Brasília; 2002.

7. Almeida MCP, Mishima SM. O desafio do trabalho em equipe na atenção à Saúde da Família: construindo novas autonomias no trabalho. Interface Comun Saúde Educ. 2001;5(9):150-3.

8. Reis CCL, Hortale VA. Programa Saúde da Família: supervisão ou "convisão"? Estudo de caso em município de médio porte. Cad Saúde Pública. 2004;20(2):492-501.

9. Ribeiro EM, Pires D, Blank VLG. A teorização sobre processo de trabalho em saúde como instrumental para análise do trabalho no Programa Saúde da Família. Cad Saúde Pública. 2004;20(2):438-46.
Entende-se que o PSF tem como propósito priorizar ações de prevenção e promoção da saúde, portanto, é fundamental que as equipes encontrem momentos para trabalhar em conjunto e colocar em prática a complementaridade e a interdependência dos diferentes trabalhos.

10. M inayo MCS, organizador. Pesquisa social: teoria, método e criatividade. 7a ed. Petrópolis: Vozes; 1997

11. Bardin L. Análise de conteúdo. 3ạ ed. Lisboa: Edições $70 ; 2004$

12. Conselho Nacional de Saúde. Resolução n. 196, de 10 de outubro de 1996. Dispõe sobre as diretrizes e normas regulamentadoras de pesquisas envolvendo seres humanos. Bioética. 1996;4(2 Supl):15-25.

13. Ferraz L, Aerts DRGC. Agente comunitário de saúde em Porto Alegre: um vendedor de saúde. Saúde Debate. 2004;28(66):68-74.

14. Silva IZQJ, Trad LAB. O trabalho em equipe no PSF: investigando a articulação técnica e a interação entre os profissionais. Interface Comun Saúde Educ. 2004/ 2005;9(16):25-38.

15. Campos GWS. Subjetividade e administração de pessoal: considerações sobre modos de gerenciar o trabaIho em equipes de saúde. In: Merhy EE, Onocko $R$, organizadores. Agir em saúde: um desafio para o público. São Paulo: Hucitec; 1997. p. 229-66.

16. Ortiz JN, Bordignon MO, Gralha RS, Fagundes S, Coradini SR. Acolhimento em Porto Alegre: um SUS de todos para todos. Porto Alegre: Prefeitura Municipal; 2004.

17. Fracolli LA, Zoboli ELCP. Descrição e análise do acolhimento: uma contribuição para o Programa de Saúde da Família. Rev Esc Enferm USP. 2004;38(2):143-51.

18. Conill EM. Políticas de atenção primária e reformas sanitárias: discutindo a avaliação a partir da análise do Programa Saúde da Família em Florianópolis, Santa Catarina, Brasil, 1994-2000. Cad Saúde Pública. 2002; 18 Supl:S191-202. 